\title{
ON PURE SUBGROUPS OF LCA GROUPS
}

\author{
D. L. ARMACOST
}

Abstract. In this note we determine the structure of those locally compact abelian (LCA) groups in which no nontrivial closed subgroup is pure and the structure of those LCA groups in which every closed subgroup is pure.

It is a well-known fact, and an easy exercise to show, that an abelian group is pure-simple (i.e. it contains no nontrivial pure subgroups) if and only if it is isomorphic to a subgroup of either the rationals $Q$ or a quasi. cyclic group $Z\left(p^{\infty}\right)$ (see [3] for definitions relating to abelian groups, and in particular [3, Exercise 7, p. 119] for the result stated here)。 At the other end of the spectrum, an abelian group has the property that all of its subgroups are pure if and only if it is an elementary group (i.e. a torsion group in which every element has square-free order). This result is stated as Exercise 12 in [6, p. 16]. It is the object of this note to investigate the corresponding properties within the class of locally compact abelian (LCA) Hausdorff topological groups.

Let us establish notation. The groups which we shall mention are the integers $Z$, the cyclic groups $Z(n)$ of order $n$, the quasicyclic groups $Z\left(p^{\infty}\right)$, the rationals $Q$ taken discrete, the real numbers $R$ with the usual topology, the $p$-adic integers $J_{p}$ and the $p$-adic numbers $F_{p}$, both with the ir usual topologies. See [5] for the definitions of all these groups. All groups will be assumed to be LCA groups. We use the symbol "@ " to denote topological isomorphism. Finally, we denote the character group of an LCA group $G$ by $\hat{G}$.

Definition. An LCA group $G$ is said to be pure-simple if and only if $G$ contains no nontrivial closed pure subgroups.

As we know, any discrete abelian group of rank 1 (i.e. a subgroup of $Q$ or of $Z\left(p^{\infty}\right)$ for some prime $p$ ) is pure-simple. The group $R$ provides

Received by the editors June 21, 1973.

AMS (MOS) subject classifications (1970). Primary 22D05; Secondary 22B05.

Key words and phrases. Closed pure subgroups, elementary groups, $p$ adic integers, $p$-adic numbers, pure-simple groups. 
another example of a pure-simple group. It follows from the description of the closed subgroups of $F_{p}$ in $[5,10.16(a)]$ that the groups $F_{p}$ and $J_{p}$ are pure-simple. Also, since any subgroup of $Q$ is pure-simple, it follows from [4, Théorème 2] that any quotient of $\hat{Q}$ by a closed subgroup (that is, any compact connected group of dimension 1 [5, 24.28]) is pure-simple as well. We shall show that the examples listed above exhaust all the possibilities for pure-simple groups. In preparation for this we shall present three lemmas.

Lemma 1. Let $G$ be pure-simple and torsion-free, and let $E(G)$ be the minimal divisible extension of $G$, topologized so that $G$ is an open subgroup of $E(G)[5,25.32(a)]$. Then $E(G)$ is also pure-simple.

Proof. It follows from [5, A.13] that $E(G)$ is also torsion-free. Let $H$ be any nonzero closed pure (and hence divisible) subgroup of $E(G)$. We show that $H$ must equal $E(G)$. From the torsion-freeness of $E(G)$ it is an easy matter to show that $H \cap G$ is a closed pure subgroup of $G$, so that either $H \cap G=\{0\}$ or $H \cap G=G$. Now the former case cannot occur, since otherwise we could construct by [3, Theorem 21.2] a proper divisible subgroup of $E(G)$ containing $G$, which would contradict the minimality of $E(G)$. Hence $H \cap G=G$, and so $G \subseteq H$. But since $H$ is divisible, we have $H=E(G)$, which completes the proof.

Our next lemma is a reformulation of an unpublished result of Robertson [8], which is useful in several contexts. Our proof is different from Robertson's.

Lemma 2 (Robertson). Let $f$ be a continuous homomorphism from $F_{p}$ into a totally disconnected LCA group $G$. Then $f$ is an open mapping onto its image.

Proof. Let $f^{*}: \hat{G} \rightarrow F_{p}$ be the adjoint of $f[5,24.37]$. Now every element of $\hat{G}$ is compact $[5,24.18]$, and so by $[5,5.14$ and 9.8$]$ every element of $\hat{G}$ is contained in a compact open subgroup. If $f^{*}$ (and hence $f$ ) is the zero map, there is nothing to prove. Otherwise, let $K$ be a compact open subgroup of $\hat{G}$ containing an element not in the kernel of $f^{*}$. Then $f^{*}(K)$ is a nontrivial compact subgroup of $F_{p}$ and is hence open in $F_{p}[5,10.16(\mathrm{a})]$. By $[5,5.29]$ the restriction of $f^{*}$ to $K$ is open onto $f^{*}(K)$. But since $K$ is open in $\hat{G}$ and $f^{*}(K)$ is open in $F_{p}$, we conclude that $f^{*}$ is an open mapping from $\hat{G}$ into $F_{p}$. Hence by $[5,24.40$ and 24.41$], f$ is open onto its image.

Lemma 3. Let $G$ be a totally disconnected, divisible, torsion-free LCA 
group. If $G$ is pure-simple, then $G$ is topologically isomorphic with either $Q$ or $F_{p}$ for some prime $p$.

Proof. With the help of the structure theorem for torsion-free divisible groups $[5,25.33]$ and the fact that direct summands are pure subgroups, we deduce that either $G \cong Q$ or $G$ is the minimal divisible extension of a product of groups $J_{p}$ for various primes $p$. If $G$ contains a copy of $J_{p}$ then by $[5, A .7]$ we may construct a continuous homomorphism $f$ from $F_{p}$ into $G$, since $G$ is divisible and $J_{p}$ is open in $F_{p}$. It is clear that $f$ must be oneone, and so we conclude from Lemma 2 that $f$ is a topological isomorphism from $F_{p}$ onto its image. Hence $G$ contains a copy of $F_{p}$, and since $G$ is pure-simple, we conclude that $G \cong F_{p}$. This completes the proof.

Theorem 1. An LCA group is pure-simple if and only if it is topologically isomorphic with one of the following: (a) a discrete group of rank 1, (b) $J_{p}$, (c) $F_{p}$, (d) $R$, (e) a compact connected group of dimension 1.

Proof. As has already been observed, all of the groups of types (a)-(e) are pure-simple. For the converse, suppose that $G$ is a pure-simple group, and let $C$ be the identity component of $G$. Since $C$ is divisible [5, 24.44(d)], it is a closed pure subgroup of $G$, and so $G$ is either connected or totally disconnected.

If $G$ is connected, we conclude from the structure theorem for connected groups $[5,9.14]$ that either $G \cong R$ or $G$ is compact and connected. By [4, Théorème 2] we can say that if $G$ is compact, the discrete torsion-free group $\hat{G}$ is also pure-simple, and so $\hat{G}$ must be a subgroup of $Q$. Hence by $[5,24.28] G$ is a compact connected group of dimension 1 . Thus a connected pure-simple group must be of type (d) or (e).

Next, as sume that $G$ is totally disconnected. If $G$ is not torsion-free, then by a theorem of Kulikov [3, 27.3], $G$ contains a nontrivial cocyclic subgroup $H$ (that is, $H$ is a subgroup of $Z\left(p^{\infty}\right)$ for some $p$ ) which is algebraically a direct summand of $G$. In particular, $H$ is pure in $G$. If $H$ has the form $Z\left(p^{n}\right)$ for some integer $n$, then $H$ is already closed, so that $G=H$, giving us case (a). If, on the other hand, $H$ has the form $Z\left(p^{\infty}\right)$, let $\bar{H}$ denote the closure of $H$ in $G$. By $[1$, Theorem 1] we can say that either $\bar{H}$ is compact and connected or that $\bar{H} \cong Z\left(p^{\infty}\right)$. Since the former is impossible we conclude that $\bar{H}$ is a closed pure subgroup of $G$, meaning that $G \cong$ $Z\left(p^{\infty}\right)$, which again leads us to case (a).

Finally, assume that $G$ is totally disconnected and torsion-free, and 
let $E(G)$ be the minimal divisible extension of $G$ as in $[5,25.32]$. From Lemma 1 we find that $E(G)$ is pure-simple, and since it is both totally disconnected and torsion-free, we invoke Lemma 3 to conclude that either $E(G) \cong Q$ or $E(G) \cong F_{p}$ for some prime $p$. The first possibility leads us to case (a), while the second leads us to cases (b) and (c). This completes the proof.

We now turn to a consideration of those LCA groups in which every closed subgroup is pure. We shall refer to an LCA group in which each nonzero element has prime order $p$ as an elementary p-group (see [2, p. 41] or [5, 25.29] for a description of these groups). As stated earlier, every subgroup, whether closed or not, of such a group is a pure subgroup. As a less trivial example, let $G$ be the direct product of the compact groups $Z(p)$ over all primes $p$. Every closed subgroup of $G$ is pure in $G$, although of course not every subgroup is. More generally, let $G$ be a local direct product (see $\left[2, p_{0} 7\right]$ or $[5,6,16]$ ) of elementary p-groups for various primes $p$. One may show that every closed subgroup of $G$ is pure in $G$ by applying Braconnier's structure theorem [2, $\left.\mathrm{p}_{0} 49\right]$ to an arbitrary closed subgroup of $G$. We now show that the most general LCA group in which every closed subgroup is pure must be of this general form.

Theorem 2. Every closed subgroup of an LCA group $G$ is pure in $G$ if and only if $G$ is topologically isomorphic to a local direct product of elemen. tary p-groups for various primes $p$.

Proof. To expedite our argument, let us say that a group in which every closed subgroup is pure possesses property $P$. As remarked above, a local direct product of elementary $p$-groups enjoys property $P$.

For the converse, let $G$ be a nontrivial LCA group having property P. Now $G$ cannot be connected. For if it were, then by [7, Theorem 2.9 (Corollary)] every closed subgroup of $G$ would also be connected, whereas it is not hard to show that this cannot occur. Since every closed subgroup of $G$ must also have property $\mathrm{P}$, we conclude that $G$ is totally disconnected. Moreover, each element of $G$ is compact, since the group of integers $Z$ does not have property $\mathrm{P}$ (see $[5,9.1]$ ). Hence both $G$ and $\hat{G}$ are totally disconnected, so we may apply Braconnier's theorem $[2, \mathrm{p} .49]$ to conclude that $G$ is a local direct product of its $p$-primary components $G_{p}$ (see $[2, \mathrm{p} .46]$ for the definition).

It now only remains to show that each poprimary component $G_{p}$ of $G$ is elementary. Since each $p$-primary component $G_{p}$ is closed in $G[2, \mathrm{p} .48]$ it is clear that each $G_{p}$ has property $\mathrm{P}$ as well. Now let $p$ be any prime for 
which $G_{p}$ is nontrivial, and let $x$ be any nonzero element of $G_{p^{*}}$ If $H$ is the closed monothetic subgroup of $G_{p}$ generated by $x$, we can conclude from $[5,25.16]$ that $H$ has the form $J_{p}$ or $Z\left(p^{n}\right)$ for some $n \geq 1$. Since $J_{p}$ does

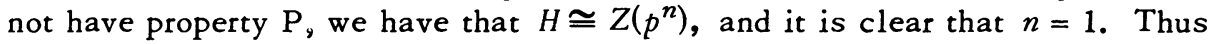
$x$ has order $p_{0}$ so $G_{p}$ is elementary, which completes the proof.

Remark. It may be shown, with some added complications, that Theorem 2 is still valid under the weaker condition that the kernel of each continuous character of $G$ is pure in $G$.

\section{REFERENCES}

1. D. Armacost and W. Armacost, On p-thetic groups, Pacific J. Math. 41 (1972), 295-301.

2. J. Braconnier, Sur les groupes topologiques localement compacts, J. Math. Pures Appl. 27 (1948), 1-85. MR 7, 114.

3. L. Fuchs, Infinite abelian groups. Vol. I, Academic Press, New York, 1970. MR $41 \# 333$.

4. S. Hartman and A. Hulanicki, Les sous-groupes pures et leurs duals, Fund. Math. 45 (1957), 71-77. MR 19, 429.

5. E. Hewitt and K. Ross, Abstract harmonic analysis。Vol. I: Structure of topological groups. Integration theory, group representations, Die Grundlehren der Math. Wissenschaften, Band 115, Academic Press, New York; Springer-Verlag, Berlin, 1963. MR 28 \#158.

6. I. Kaplansky, Infinite abelian groups, Univ. of Michigan Press, Ann Arbor, 1954. MR 16, 444.

7. M. Moskowitz, Homological algebra in locally compact abelian groups, Trans. Amer. Math. Soc. 127 (1967), 361-404. MR 35 \#5861.

8. L. Robertson, Transfinite torsion, poconstituents, and splitting in locally compact abelian groups (unpublished).

DEPARTMENT OF MATHEMATICS, AMHERST COLLEGE, AMHERST, MASSACHUSETTS 01002 